\title{
Mecanismos evaluables con dinámica de sistemas, utilizables en desarrollo de software libre para gestión de cooperación
}

\author{
Evaluable Mechanisms by Sustem Dunamics, Usable in Free \\ Software Development for the Cooperation Management
}

\section{Resumen}

Presenta una revisión de la literatura sobre los mecanismos de cooperación utilizables en la gestión de la cooperación en proyectos de desarrollo de software libre, así como las bases teóricas acerca de la cooperación, y la posibilidad de su estudio mediante dinámica de sistemas. $\mathrm{Se}$ concluye que se requieren lineamientos y estrategias aplicables a metodologías de desarrollo de software, para realizar una gestión efectiva de la cooperación y así evitar los dilemas sociales que pueden afectar la producción de este tipo de software.

Palabras clave: Software libre, Dinámica de sistemas, Mecanismos de cooperación, Metodologías de desarrollo de software.

\section{Abstract}

This is a literature review about the cooperation mechanism applied to manage the collective action in the free software development process, and the theoretical foundations about cooperation, plus the possibility to apply the system dynamics. It was concluded that guidelines and strategies are required to promote effective cooperation in free software development process, in order to solve the social dilemmas which could affect the production of this kind of systems.

Keywords: Free Software, System Dynamics, Open Source, Cooperation Mechanisms, Software Development Methodology.

* M.Sc. Universidad Autónoma de Bucaramanga (Colombia). 1villamizar5@unab.edu.co

** Ph.D. Universidad Autónoma de Bucaramanga (Colombia). andrick.parra@gmail.com 


\section{INTRODUCCIÓN}

En las comunidades suelen aparecer dilemas sociales, debido a que el individuo tiende a realizar sus actividades buscando un beneficio propio, sin tener en cuenta el bienestar colectivo y sin comprender que si todos los miembros cooperan se podrán evitar tales dilemas y obtener mejores resultados; así, un dilema social se entiende como la tensión entre la racionalidad individual y el bienestar colectivo [1]. La comunidad de desarrollo de software libre no está exenta de estos dilemas sociales, a pesar de que es conocida por manejar su desarrollo por medio del trabajo colaborativo, que sigue un modelo llamado Bazar, en el cual se realizan aportes de todos los interesados en colaborar, y una vez se tiene un programa ejecutable se libera la versión [2]. En muchos casos esto ha funcionado hasta llegar a proyectos exitosos; pero en ocasiones los prototipos llevan código dañino, que ocasiona muchos errores; el código hace referencia al bien público que se comparte. $\mathrm{Si}$ esta comunidad tuviese un método formal que todos sus integrantes siguieran, sería posible realizar un mejor seguimiento $\mathrm{y}$, así, liberar versiones con menos errores.
El software libre es particularmente sensible a la cooperación; si no aparece la cooperación en niveles suficientes, pudiera no proveerse en cantidad suficiente el software libre. Así, se justifica realizar una revisión de la literatura que permita identificar los mecanismos de cooperación que los desarrolladores de software utilizan para enfrentar los dilemas sociales, que pudieran incluso impedir el desarrollo del software libre o incluso reducir su disponibilidad.

Conocidos los mecanismos, se propone revisar si la Dinámica de Sistemas ha sido utilizada para definir estrategias y lineamientos aplicables al desarrollo de software libre que promuevan la cooperación en su desarrollo.

\section{Condiciones de LA REVISIÓN}

Inicialmente, se delimitaron los aspectos claves para realizar la búsqueda de la literatura. Estas condiciones van desde las bases de datos utilizadas, hasta las fechas seleccionadas para realizar la búsqueda. Las condiciones planteadas se pueden observar en la tabla 1.

\section{Tabla 1}

\section{CONDICIONES DE BÚSQUEDA DE LA REVISIÓN DE LA LITERATURA}

\begin{tabular}{|c|c|}
\hline Condición & Descripción \\
\hline $\begin{aligned} \text { O Bases de datos: } \\
\text { - } \\
\text { - } \text { EBoquest } \\
\text { - Scopus } \\
\text { - } \text { Google scholar }\end{aligned}$ & Bases de datos donde se realizaron las búsquedas. \\
\hline $\begin{aligned} \text { O Fechas: } & \\
& \text { - Documentos del año } 2000 \text { hasta el } \\
& \text { presente. } \\
& \text { Documentos relevantes para el proyecto. }\end{aligned}$ & $\begin{array}{l}\text { Característica con las que se delimitaron las fechas de } \\
\text { los documentos que se seleccionaron: del año } 2000 \text { a la } \\
\text { actualidad, pero también documentos con fecha fuera de } \\
\text { este lapso que contienen información relevante para el } \\
\text { proyecto. }\end{array}$ \\
\hline - Trabajos o artículos más citados & $\begin{array}{l}\text { Se tuvo en cuenta el número de citaciones de los } \\
\text { documentos. }\end{array}$ \\
\hline ○ Trabajos en español o en inglés & $\begin{array}{l}\text { Por medio de esta condición se define que se tendrán en } \\
\text { cuenta únicamente documentos en estos dos idiomas. }\end{array}$ \\
\hline
\end{tabular}


Para la búsqueda de los documentos también se identificaron unas palabras claves, que permiten hacer una búsqueda más precisa. Estas palabras claves son:

- Cooperación

- Software Libre

- Dilemas Sociales

- Dinámica de Sistemas

La Fig. 1 sintetiza la estructura que ordena el área de investigación revisada.

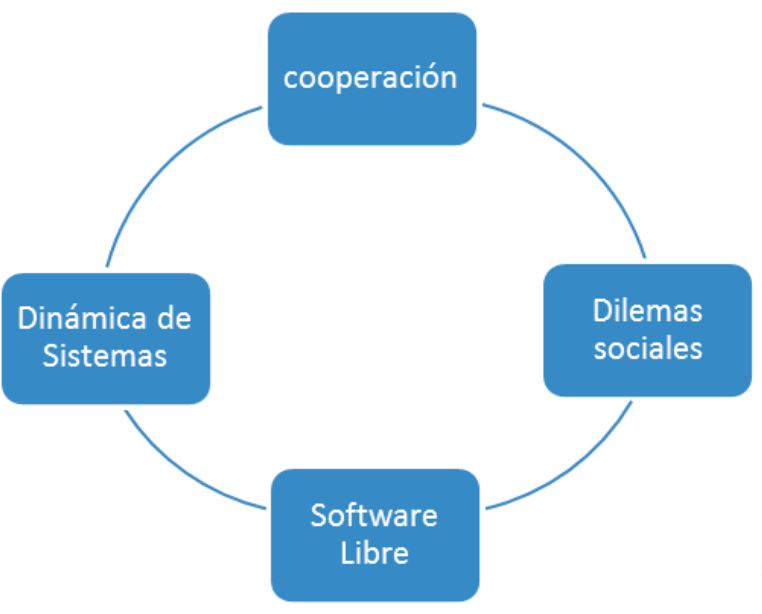

Fig. 1 Primera aproximación a una estructura de revisión que representa el área de investigación definida por la cooperación, los dilemas sociales y la dinámica de sistemas.

En la Fig. 2 se presenta una segunda versión de estructura de revisión para el área; en ella se presentan las subáreas en las que se ordena la revisión. Para la Cooperación se resaltaron las bases teóricas acerca de la cooperación y los dilemas sociales; para la Dinámica de Sistemas se hizo énfasis en sus posibilidades como método para evaluar una estrategia de gestión de la cooperación en términos genéricos; en cuanto al Software Libre, se hizo énfasis en las metodologías de desarrollo y en las posibilidades que ofrecen a la aplicación de mecanismos de cooperación. Luego de presentada la estructura general de la revisión, se procede a describir los antecedentes encontrados.

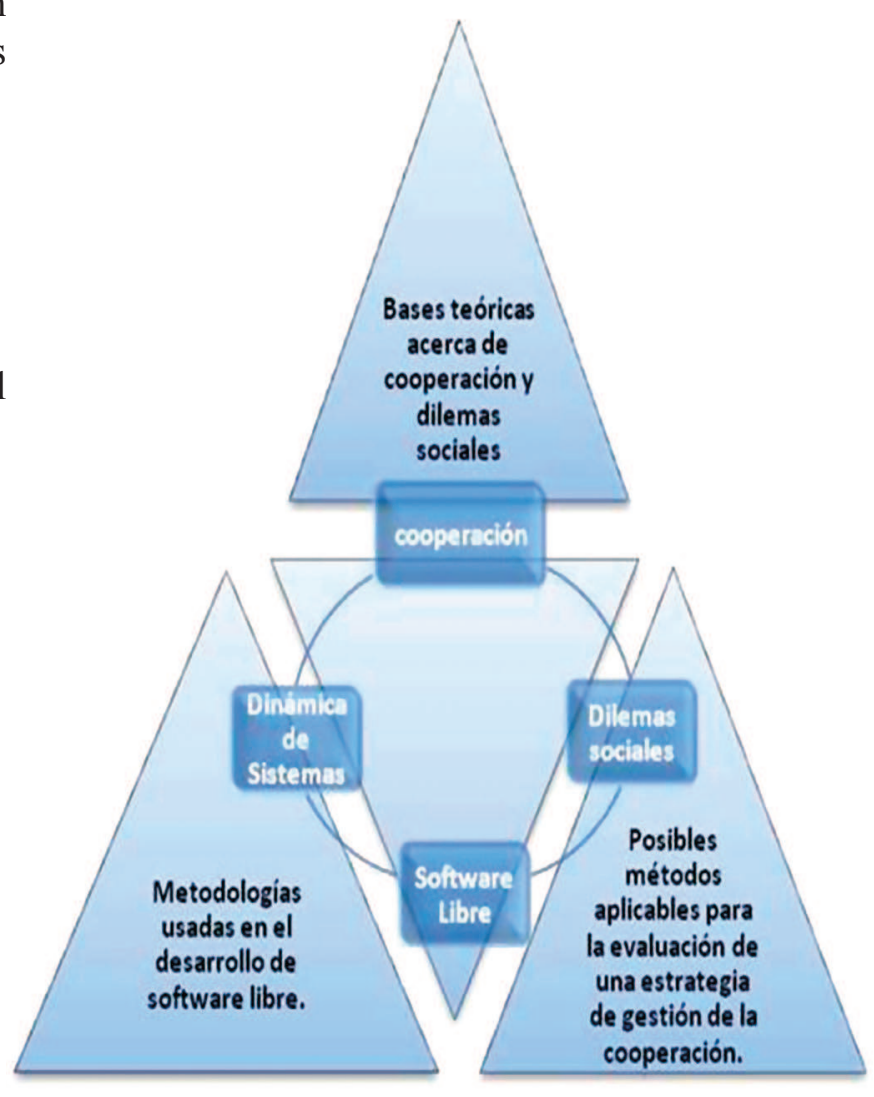

Fig. 2 Ubicación del proyecto en la clasificación del estado del arte, segunda versión.

\section{DESCRIPCIÓN DEL TRABAJO}

La revisión de la literatura se encuentra dividida en cuatro secciones, que permiten dar base a la propuesta de una estrategia de gestión de cooperación que permita mejorar la gestión y planificación de los proyectos de software libre y cómo los desarrolladores promueven y sostienen la cooperación. Estas secciones son: Bases teóricas acerca de cooperación y dilemas sociales, Posibles métodos aplicables para la evaluación de una estrategia de gestión de la cooperación, Metodologías usadas en el desarrollo de software libre e Impacto del software libre en la sociedad. 


\section{A. Bases teóricas acerca de Cooperación y Dilemas Sociales}

Inicialmente hay que anotar que un dilema social consiste en el enfrentamiento entre la racionalidad individual y el bienestar colectivo; esta situación conduce, en muchas ocasiones, a una situación peor que la situación inicial. Los dilemas sociales pueden contener gran cantidad de variables que al ser afectadas pueden causar grandes efectos [1]. Esta situación de dilema social ocurre porque los individuos piensan en su bienestar individual sin importar los efectos que puedan tener sobre los demás individuos, es decir, tienen un pensamiento egoísta que hace que piensen en su propio beneficio. Los dilemas sociales terminan por no beneficiar a nadie [1].

Los dilemas sociales han sido objeto de estudios desde la economía, la ciencia política, la psicología y la ingeniería de sistemas. Una de las expresiones más importantes de este tipo de situaciones fue sintetizada en la tragedia del terreno común [3]. Plantea un dilema de cómo los individuos motivados por un interés individual llegan a actuar de forma independiente, y esto hace que no compartan los recursos como bienes comunes, hasta el punto de llegar a destruir estos recursos $\mathrm{y}$, así, ninguno llega a beneficiarse de estos; esta situación es un ejemplo de dilema social, ya que existe un conflicto entre los beneficios de los individuos y el bienestar colectivo en cuanto a la explotación de los recursos comunes. En este documento se planteó que la única solución es tener un agente externo que permita supervisar $\mathrm{y}$ hacer que los individuos lleguen a acuerdos acerca del uso de los recursos comunes, ya que se considera que estos no son capaces de llegar a algún acuerdo por sí solos.

En un grupo, a pesar de los intereses comunes $\mathrm{y}$ de que se pueda llegar a un acuerdo para resolver mediante la cooperación una situación y todos puedan beneficiarse, existe el riesgo de que aparezcan individuos que pretendan aprovecharse y sacar ventajas para su beneficio propio y llegar a que la mayoría explote a una minoría. Se pude dar una acción colectica en un grupo, pero también en determinado momento aparecerán los llamados "free rider", que buscan obtener beneficios sin cooperar con los demás individuos y aprovecharse de los esfuerzos de los demás, que sí trabajan con la comunidad para hacer buen uso de los recursos comunes. Los bienes públicos pueden ser usados por cualquier individuo, y no hay forma de impedirlo, y a pesar de que una comunidad tenga intereses comunes, se asume que los seres humanos racionalmente no deciden cooperar, ya que siempre van a buscar su beneficio propio, piensan de forma egoísta. Aunque existe la posibilidad de que pueda darse una acción colectiva que llegue a ser exitosa, pero solo si la minoría llegara a dominar a la mayoría [4].

E. Ostrom [5] tratatemas comolaacción colectiva, con la cual es posible solucionar los dilemas sociales. La acción colectiva se ha considerado una opción para solucionar los dilemas sociales, pero debido a la forma egoísta de pensar del individuo en ocasiones no tiene resultados positivos; tampoco esta acción colectiva es posible porque en una comunidad una mayoría trata de aprovecharse de una minoría; pero daría una solución completa si todos contribuyeran. También en una acción colectiva aparece una tentación del individuo a retirarse y pasar de los que contribuyen a los que no lo hacen, debido a que no ven necesario cooperar, porque los demás no responden con una acción cooperativa igual. También se plantea que para que exista una cooperación exitosa es importante que exista una comunicación cara a cara entre los individuos, para que se genere una confianza de cooperación y posteriormente aclarar una estrategia que permita llegar a resultados óptimos. Por medio de la comunicación cara a cara se crea una memoria que permite ir creando una reputación que permitirá llegar a una confianza, que hace que cada individuo decida cooperar. 
En los documentos que se encuentran acerca de estudios de dilemas sociales se tratan dilemas sociales de pequeña escala; en estos documentos se plantea cómo evaluar distintos mecanismos de cooperación, donde se busca promover la cooperación para llegar a una solución a estos dilemas sociales de pequeña escala. J. A. Parra [6] se planteó un constructo que pueda evaluar mecanismos para dilemas sociales de gran escala; en este se integraron tres mecanismos: basado en confianza, por percepción de daño y por norma. También en este trabajo se realizaron una serie de modelos en dinámica de sistemas acerca de unos casos hipotéticos, donde lo que se pretendía era evaluar la efectividad de los mecanismos. En este trabajo se explica cómo estos mecanismos pueden llegar a promover la cooperación para dar solución a dilemas sociales de gran escala.

En el libro "Constructo para la evaluación de la cooperación en dilemas sociales de gran escala" [6] se identifican una serie de aspectos problemáticos; uno es que cuando los individuos se ven envueltos un dilema social la decisión es de retirarse normalmente, y otra es que si ellos toman la decisión de cooperar, deciden cuándo lo hacen y con qué frecuencia; también se exponen tipos de dilemas sociales de acuerdo con el bien económico. En la tabla 2 se pueden observar los tipos de dilemas sociales.

TABLA 2

TIPOS DE DILEMAS SOCIALES DE ACUERDO CON EL BIEN ECONÓMICO SUSCEPTIBLE, SU LOCALIZACIÓN Y MANIFESTACIONES [7-9]

\begin{tabular}{|l|l|l|}
\hline \multicolumn{1}{|c|}{ Tipo de bien } & \multicolumn{1}{|c|}{$\begin{array}{c}\text { Proceso en el que ocurre el } \\
\text { dilema }\end{array}$} & \multicolumn{1}{c|}{ Manifestaciones } \\
\hline Bien público & Provisión & Subprovisión \\
\hline Recurso común & Apropiación & $\begin{array}{l}\text { Congestión, contaminación } \\
\text { sobreutilización. }\end{array}$ \\
\hline
\end{tabular}

Fuente: "Constructo para la evaluación de la cooperación en dilemas sociales de gran escala" [6]

Existen dilemas sociales de pequeña y gran escala; en la tabla 3 se expone un comparativo de las diferentes características de estos [6], basado en la referencia [10]. Esta tabla permite establecer las diferencias entre estos dos dilemas sociales, para así identificar en cuál podría ubicarse el planteamiento de una estrategia de gestión de la cooperación.

\section{TABLA 3}

CARACTERIZACión de los dilemas SOCIALES DE PEQUeÑa Y GRAN ESCALA $[10,11]$

\begin{tabular}{|l|l|l|}
\hline \multicolumn{1}{|c|}{ Tipo } & \multicolumn{1}{c|}{ Pequeña escala } & \multicolumn{1}{c|}{ Gran escala } \\
\hline Contexto & Campo y laboratorio & Difícil de localizar \\
\hline Tamaño grupo & Menor 10 & Más de 10 \\
\hline Características grupo & Homogéneas & Heterogéneas \\
\hline Magnitud retardo & Pocos minutos & Alta, días, meses o años \\
\hline Calidad realimentación & Alta & Baja \\
\hline Modelo racionalidad & Acotado & Acotado \\
\hline Encuentros & Uno, finitos & Infinitos \\
\hline Comunicación & Frente a frente & Medida medios masivos \\
\hline
\end{tabular}

Fuente: "Constructo para la evaluación de la cooperación en dilemas sociales de gran escala" [6] 
Se asumía que los seres humanos, racionalmente, no deciden cooperar, ya que buscan un beneficio propio; aunque podría darse una acción colectiva que llegue a ser exitosa, pero eso solo sucederá si la minoría pudiera llegar a dominar la mayoría [4]. Por el contrario, en la referencia [5] se expone que la cooperación es posible si se construye una confianza y reputación de cooperación; esto está expuesto en la teoría de la cooperación. En la tabla 4 se puede observar un comparativo de la teoría de la no cooperación y de la cooperación.

TABLA 4

SÍNTESIS DE LA REVISIÓN COMPRENSIVA DE LA LITERATURA PARA LA TEORÍA COOPERATIVA Y NO COOPERATIVA DE DILEMAS SOCIALES [8]

\begin{tabular}{|c|c|c|}
\hline & Teoría no Cooperativa & Teoría Cooperativa \\
\hline Características & $\begin{array}{l}\text { - } \\
\text { - } \quad \text { Inforfecta racionalidad } \\
\text { - Un encuentro o encuentros } \\
\text { finitos }\end{array}$ & $\begin{array}{ll}\text { - } & \text { Racionalidad limitada } \\
\text { - } & \text { Información retardada y } \\
\text { distorsionada } \\
\text { - } \\
\text { - } \quad \text { Darios encuentros repetidos. } \\
\text { iniciales }\end{array}$ \\
\hline Recomendaciones políticas & $\begin{array}{l}\text { - } \quad \text { Control por un agente externo } \\
\text { - } \\
\text { prignación de derechos de } \\
\text { propiedad privada }\end{array}$ & Cooperación \\
\hline Referencias seleccionadas & {$[3,10,12,13,14]$} & {$[8,10]$} \\
\hline
\end{tabular}

Fuente: "Constructo para la evaluación de la cooperación en dilemas sociales de gran escala" [6].

En la referencia [6] se identifican tres formas con las que los grupos humanos pueden enfrentar los dilemas sociales:

- Asignación de derechos de propiedad privada [15]

- Control por un agente externo [3]

- Cooperación como autorregulación [10]

Anteriormente se ha hablado de tipos de bienes, que son:

- Bienes privados: presentan exclusividad; poseen rivalidad, porque no pueden ser usados al mismo tiempo ni por todos los usuarios, y esto disminuye la posibilidad de ser usados por otros. Se puede excluir usuarios [16].

- Bienes de club: presentan exclusividad, pero no poseen rivalidad, ya que no se impide el uso por otros y pueden ser usados al mismo tiempo [16].

- Bienes públicos: no son exclusivos ni poseen rivalidad; pueden ser usados por otros, y si una persona lo usa esto no impide que sea usado por otro [7].

- Recursos comunes: no son bienes exclusivos, pero sí poseen una rivalidad, ya que a mediad que son usados reducen la posibilidad de ser usados por otros, pero es difícil excluir usuarios [7].

Entonces, los bienes públicos están disponibles para todos, y si son usados por una persona esto no impide que sean usados por otros; estos bienes no impiden su uso simultáneo, es decir, es no rival y no excluyente. Por su parte, los recursos comunes, al ser usados por una persona, disminuyen el uso por otros, pero al mismo tiempo es difícil excluir usuarios; estos bienes pueden ser naturales o elaborados por alguien [7].

En la tabla 5 se presenta un cuadro con los diferentes tipos de bienes económicos, clasificados según el consumo. 
TABla 5

TIPOS DE BIENES ECONÓMICOS

\begin{tabular}{|l|l|l|}
\hline \multicolumn{1}{|c|}{ Bienes } & \multicolumn{1}{|c|}{ Consumo no excluible } & \multicolumn{1}{c|}{ Consumo excluible } \\
\hline Consumo no rival & $\begin{array}{l}\text { Bienes Públicos Puros (ej. } \\
\text { Defensa, semáforos) }\end{array}$ & Bienes de Club (ej. TV cable) \\
\hline Consumo rival & $\begin{array}{l}\text { Bienes en Común (ej. bancos de } \\
\text { pesca) }\end{array}$ & Bienes Privados \\
\hline
\end{tabular}

Fuente: “Gobernar el mercado. Las nuevas fronteras de Estado en el siglo XXI” [16]

Un dilema social puede darse entre un grupo (de dos individuos en adelante), ya que se empieza a observar un enfrentamiento entre lo que cada individuo quiere, y esto tenderá a que cada uno busque su beneficio propio. Para estabilizar esta situación se propone enfrentarla por medio de cooperación. La cooperación es compleja y se puede relacionar con dos aspectos específicos: la dependencia a las condiciones iniciales y los retardos de información [17]; además, para que esta se dé debe existir una confianza de cooperación que hace que cada uno de los miembros decidan cooperar. Si hay mayor cooperación habrá más confianza en la cooperación futura, y así se podrá promover y sostener la cooperación [10]. En la Fig. 3 se expone la versión dinámica del mecanismo de cooperación basado en confianza [17].

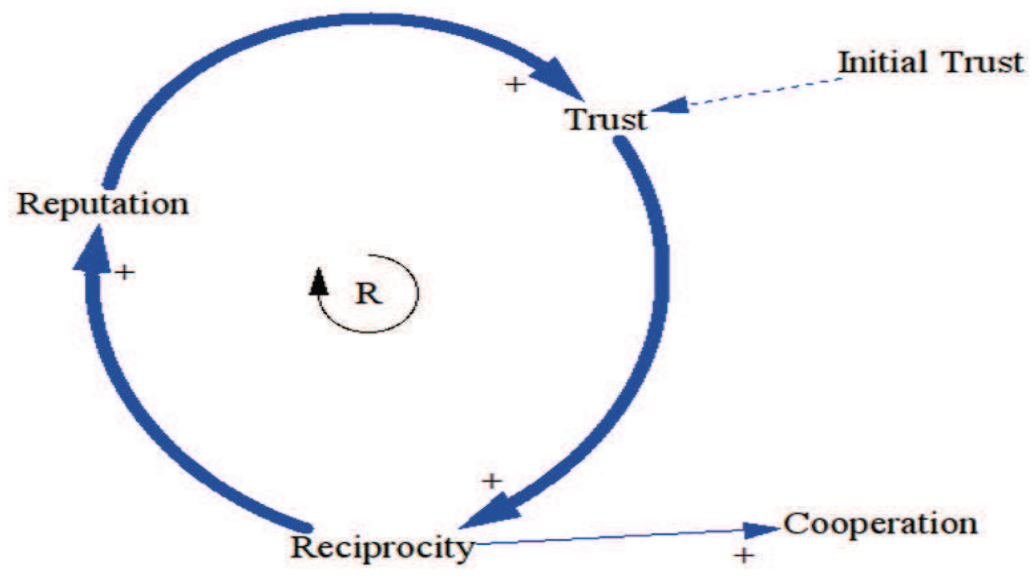

Fig. 3 Versión dinámica del mecanismo de Cooperación Basado en Confianza. Fuente: “Gestión de la cooperación en dilemas sociales: un aporte desde la ingeniería de sistemas" [17].

B. Posibles métodos aplicables para la evaluación de una estrategia de Gestión de la Cooperación

Existen unas herramientas que se podrían aplicar para desarrollar un modelo que permita integrar y evaluar una estrategia de gestión de la cooperación diseñada; estas son: Teoría de Juegos, Agentes, Autómatas celulares y Dinámica de sistemas; la tabla 6 muestra una comparación de las diferentes características de estas herramientas. 
TABLA 6

HERRAMIENTAS QUE SE PODRÍAN APLICAR AL PROYECTO

\begin{tabular}{|c|c|c|}
\hline Herramienta & Características generales & $\begin{array}{c}\text { Características para ser aplicada en el } \\
\text { proyecto }\end{array}$ \\
\hline Agentes & $\begin{array}{l}\text { - El fenómeno que se modela se asume como un } \\
\text { sistema constituido por agentes [18]. } \\
\text { - Los agentes interactúan por medio de reglas de } \\
\text { decisión [18]. } \\
\text { - Cada agente está integrado por un programa } \\
\text { que contiene datos y reglas de comportamiento } \\
\text { que operan sobre los datos[18]. } \\
\text { - Se pueden implementar en cualquier lenguaje } \\
\text { de programación, pero preferiblemente que } \\
\text { sea orientado a objetos. } \\
\text { - Permite representar la provisión y apropiación } \\
\text { de algunos recursos comunes [19]. }\end{array}$ & $\begin{array}{l}\text { - Permite representar situaciones complejas, pero } \\
\text { su explicación no es directa [20]. } \\
\text { - Describe el comportamiento individual e un } \\
\text { agente, pero no se pude especificar y evaluar } \\
\text { reglas de comportamiento agregado [21]. }\end{array}$ \\
\hline $\begin{array}{l}\text { Autómatas } \\
\text { celulares }\end{array}$ & $\begin{array}{l}\text { - Modelo que integra un sustrato infinito de } \\
\text { celdas contiguas, que pueden contener un } \\
\text { conjunto finito de estados [22]. } \\
\text { - Las reglas (sencillas) definen el cambio de las } \\
\text { celdas, pero pueden generar comportamientos } \\
\text { complejos [22]. } \\
\text { - Aplicada en recursos comunes, donde se } \\
\text { estudia el comportamiento colectivo [23]. } \\
\text { - Se basa en una posición con el individualismo } \\
\text { metodológico, pone como unidad fundamental } \\
\text { el individuo [20]. }\end{array}$ & $\begin{array}{l}\text { - La realimentación y circularidad son cubiertas } \\
\text { parcialmente [20]. } \\
\text { - Se puede hacer una representación por medio } \\
\text { de reglas donde se puedan implementar } \\
\text { retardos de información y percepción para } \\
\text { individuos [24]. } \\
\text { - No se pude representar comportamientos } \\
\text { promedio [24]. } \\
\text { - Dificulta la explicación causal del } \\
\text { comportamiento [20]. }\end{array}$ \\
\hline $\begin{array}{l}\text { Teoría } \\
\text { juegos }\end{array}$ & $\begin{array}{l}\text { - Estudia la toma de decisiones, en donde } \\
\text { se puede percibir cómo los que toman las } \\
\text { decisiones intentan manipular el ambiente, y } \\
\text { cómo este los intenta manipular a ellos [25]. } \\
\text { - Los jugadores toman decisiones de un número } \\
\text { limitado de opciones [26]. } \\
\text { - Dependiendo de lo que selecciones se obtiene } \\
\text { un castigo o un premio [27]. } \\
\text { - Se usa en biología y en psicología [7]. } \\
\text { - Lo que se pretende es que cada jugador } \\
\text { maximice sus ventajas por encima de los } \\
\text { demás [28]. }\end{array}$ & $\begin{array}{l}\text { - Considera una representación específica de } \\
\text { cada opción de decisión de cada jugador [25]. } \\
\text { - Dificultad para evaluar la efectividad e } \\
\text { mecanismos de cooperación en grupos } \\
\text { numerosos [29]. } \\
\text { - No hay claridad acerca de los problemas de } \\
\text { percepción inadecuada de la realimentación y } \\
\text { como pueda afectar en las decisiones dinámicas } \\
\text { [30,31]. } \\
\text { - No ofrece de manera directa una explicación } \\
\text { causal acerca de las decisiones que se tomaron } \\
\text { - } 32] \text {. } \\
\text { No permite una representación promedio de la } \\
\text { toma decisiones [33]. }\end{array}$ \\
\hline $\begin{array}{l}\text { Dinámica de } \\
\text { sistemas }\end{array}$ & $\begin{array}{l}\text { - Es un enfoque para el análisis de políticas y } \\
\text { el diseño asistido por computador; permite } \\
\text { aplicar problemas dinámicos en sistemas } \\
\text { sociales, económicos y ecológicos [34]. } \\
\text { - Se asume como un proceso de construcción } \\
\text { incremental de confianza [35]. }\end{array}$ & $\begin{array}{l}\text { - Permite representar la realimentación y la } \\
\text { circularidad [31,33,36]. } \\
\text { - Permite especificar retardos de información [37]. } \\
\text { - Tiene estructuras que permiten describir el } \\
\text { proceso de percepción de información [38]. } \\
\text { - Se puede representar el procesamiento promedio } \\
\text { y agregado de la información [39]. } \\
\text { - Permite mostrar el comportamiento generado } \\
\text { por las condiciones iniciales y la estructura [35, } \\
\text { 40]. } \\
\text { - Permite especificar procesos de toma de } \\
\text { decisiones dinámicas [39,41]. }\end{array}$ \\
\hline
\end{tabular}

Fuente: "Constructo para la evaluación de la cooperación en dilemas sociales de gran escala" [6] 
Una vez realizada la revisión de cada una de estas herramientas, se seleccionó Dinámica de sistemas, ya que es la más completa y la que más se adecúa. Lo que se quiere realizar es la propuesta de una estrategia que posteriormente se pueda evaluar mediante un modelo por medio de la dinámica de sistemas, que, como se puede observar en el cuadro de herramientas, permite realizar el modelamiento e ir haciendo un seguimiento del comportamiento del proceso, $\mathrm{y}$ debido a que se pueden tener en cuenta retardos en la información y revisar qué sucedió en el proceso de toma de decisiones, teniendo en cuenta sus condiciones iniciales. Se puede revisar cómo sería el comportamiento según las condiciones iniciales, como la confianza de cooperación, si se aumenta esta variable de cooperación. Las demás herramientas presentan debilidades que podrían dejar inconclusas las reacciones en el comportamiento del proceso.

Para mayor detalle, a continuación se expone cómo es el método aplicado con la dinámica de sistemas [41]; los pasos que se siguen son los siguientes:

- Articular el problema

- Desarrollar hipótesis dinámica que explique la causa del problema

- Construir un modelo de simulación por computador

- Evaluar si el modelo reproduce el comportamiento observado en el mundo real

- Diseñar y evaluar políticas para aliviar el problema

- Implementar la solución

\section{Metodologías usadas en el desarrollo de software libre}

En la referencia [42] se explica cómo surge la necesidad de crear una comunidad donde cada uno de sus miembros pudiera acceder libremente al código, es decir, que este fuese libre, abierto y se pudiera usar y adaptar a nuestras necesidades.
Anteriormente, si en los grupos de desarrollo se compartía el código se debía firmar un acuerdo de confidencialidad, y si se llegaba a compartir se consideraba piratería. Gracias a la aparición de esta comunidad han surgido proyectos que han sido exitosos y han beneficiado a la comunidad. Lo que la comunidad quiere es que cada uno de estos proyectos sea compartidos, y así aportar cada vez más a la comunidad. También expresa que "la cooperación con los demás contribuye a la base de la sociedad". Es por eso que una de las grandes preocupaciones es que algunas personas acceden a este código libre, realizan sus proyectos y no vuelven a compartir con la comunidad, haciendo que su producto termine como un producto propietario, lo cual estaría violando las libertades del desarrollo de software libre y no estaría beneficiando a toda la comunidad. Estas libertades son:

- "Libertad de ejecutar el programa sea cual sea el propósito"

- "Libertad para modificar el programa para ajustarlo a tus necesidades"

- "Libertad de redistribuir copias, ya sea de forma gratuita, ya sea a cambio del pago de un precio"

- "Libertad de distribuir versiones modificadas del programa, de tal forma que la comunidad pueda aprovechar las mejoras introducidas"

En el documento "Open Source - New Rules in Software Development" [43] se expresa que el software libre permite que el usuario pueda acceder a los derechos de leer y manipular el código según sus necesidades. También hay dos actores diferentes que son los desarrolladores de software libre, los cuales hacen pequeñas o grandes contribuciones. También están las firmas o empresas que usan el código que desarrollan los primeros actores y los ponen en uso según sus necesidades. También se explica el software libre como un dilema social; el código hace referencia a un bien público. Los dilemas sociales generalmente se asocian a dos problemas: el 
sobreuso y la poca provisión o poca cooperación; en el caso del software libre no se habla de un problema de sobreuso, ya que esto produciría efectos favorables. En este caso no es excluyente que los beneficios individuales tampoco coinciden con los de toda la comunidad. La comunidad de software libre está basada en normas y confianza.

Hoy en día la Internet es un elemento importante para que lo desarrolladores compartan el trabajo realizado, y esto se puede realizar por un bajo costo. En los grupos de desarrollo se encontraron tres características importantes: estos grupos no tienen ni pasado ni futuro común, son grupos geográficamente dispersos y se comunican vía electrónica.

Acerca de la confianza se pueden encontrar dos niveles; el primero hace referencia al desarrollo del bien público, que es el código abierto; es importante que haya confianza mutua, ya que los involucrados esperan no encontrar a nadie que quiere obtener beneficios sin hacer ningún esfuerzo, es decir, no esperan encontrar al llamado "free-rider". El segundo nivel hace referencia al dilema social que se presenta en esta comunidad, que expresa que es importante establecer normas de cooperación que permitan que los individuos puedan incentivarse y así cooperar y poder contribuir al desarrollo del bien público, en este caso el código.

El documento "Trust and Community in Open Source Software Production" [44] explica cómo el rol de la confianza juega un papel importante en las comunidades de desarrollo de software libre, ya que debe haber una motivación fuerte a contribuir al desarrollo del bien público que es el código. En esta comunidad también se presenta un dilema social, y este ha sido ubicado en unos niveles; el primer nivel es el "free riding"; el segundo es acerca de las reglas del juego para prevenir que aparezca el "free riding". Se detectaron un par de antecedentes; el primero, las instituciones deben crearse con la seguridad de que la motivación de los que confían no será destruida; el segundo, si los individuos no cooperan o no contribuyen al bien público, el costo de no hacerlo será alto.

En este documento también se pueden encontrar una serie de tipos de contribuyentes al software libre; a continuación se encuentran los tipos de contribuyentes (tabla 7):

TABLA 7

CONTRIBUYENTES AL SOFTWARE LIBRE

\begin{tabular}{|l|l|}
\hline \multicolumn{1}{|c|}{ Contribuyente } & \multicolumn{1}{c|}{ Característica } \\
\hline Proveedor de servicios comerciales & $\begin{array}{l}\text { Buscan obtener dinero por medio del software libre desde el punto que } \\
\text { esto es un bien público. }\end{array}$ \\
\hline Personalizadores de software & $\begin{array}{l}\text { Estos pueden llegar a obtener un beneficio no monetario por adaptar } \\
\text { el software a sus propios beneficios. Estos solo esperan un beneficio } \\
\text { personal. }\end{array}$ \\
\hline Inversores de reputación & $\begin{array}{l}\text { Pueden obtener dinero de una forma indirecta, ya que tienen una alta } \\
\text { capacidad para el software libre, y esta se puede convertir en dinero por } \\
\text { medio del empleo de una compañía comercial. }\end{array}$ \\
\hline "Homo Ludens" & $\begin{array}{l}\text { Este grupo hace contribuciones al desarrollo del software libre casi sin } \\
\text { esperar una remuneración, ya que lo hacen "solo por diversión". }\end{array}$ \\
\hline Miembros de la tribu & $\begin{array}{l}\text { Después de realizar sus aportes no reciben beneficios tangibles, pero } \\
\text { sí beneficios psicológicos, ya que sienten satisfacción y motivación } \\
\text { ayudando a otros. }\end{array}$ \\
\hline
\end{tabular}

Fuente: "Trust and Community in Open Source Software Production" [44] 
Según la referencia [45], el principal modelo de desarrollo de software libre es el modelo Bazar. También existe un modelo llamado Catedral [46], que consiste en tener al mando a una persona y tener una jerarquía para que el proyecto funcione. Por el contrario el modelo Bazar lo que busca es que se realicen constantes avances, que se vayan liberando rápido, distribuir las responsabilidad y las tareas y finalmente estar muy abiertos esperando cooperación y aportes de todos los que deseen participar. En la tabla 8 se presenta un comparativo entre estos dos modelos.

\section{TABLA 8}

Modelo Bazar vs. modelo Catedral

\begin{tabular}{|l|l|}
\hline \multicolumn{1}{|c|}{ Bazar } & \multicolumn{1}{|c|}{ Catedral } \\
\hline $\begin{array}{l}\text { En este modelo hay un grupo desarrollando, sin tener una } \\
\text { jerarquía presente. }\end{array}$ & $\begin{array}{l}\text { Nombre adoptado por tener un enfoque más planeado } \\
\text { y centralizado. }\end{array}$ \\
\hline $\begin{array}{l}\text { Es un trabajo colaborativo, por esta razón es abierto; } \\
\text { esto permite que se puedan recibir aportes de personas } \\
\text { externas al proyecto. Aunque carece de mecanismos de } \\
\text { control de calidad. }\end{array}$ & $\begin{array}{l}\text { El encargado de administrar el proyecto debe tomar } \\
\text { decisiones acerca de su dirección y ejecución. }\end{array}$ \\
\hline $\begin{array}{l}\text { Se liberan versiones de forma rápida, las que más se } \\
\text { pueda. }\end{array}$ & $\begin{array}{l}\text { Este modelo expone una serie de lineamientos y } \\
\text { características de los proyectos de desarrollo de } \\
\text { software libre. }\end{array}$ \\
\hline $\begin{array}{l}\text { En el momento que se libera una versión no importa si } \\
\text { tiene errores. Lo importante es liberar una versión. }\end{array}$ & $\begin{array}{l}\text { Se tiene un grupo pequeño para realizar pruebas y } \\
\text { encontrar fallas o defectos. }\end{array}$ \\
\hline $\begin{array}{l}\text { Las pruebas las realizan miles de personas que desean } \\
\text { probar las versiones liberadas sin tener que pagar por esto. }\end{array}$ & $\begin{array}{l}\text { Las responsabilidades del administrador del proyecto } \\
\text { son: } \\
\text { Definir objetivos, Monitorear detalles, Motivar } \\
\text { personas, Organizar y Asignar recursos }\end{array}$ \\
\hline $\begin{array}{l}\text { Este modelo tiene los siguientes principios: "Libera a } \\
\text { menudo, libera rápido" y "Dados suficientes ojos, todo } \\
\text { error es superficial". }\end{array}$ & $\begin{array}{l}\text { Este modelo tiene las siguientes etapas: } \\
\text { Análisis de requisitos, Diseño ,Implementación, } \\
\text { Pruebas y } \\
\text { Mantenimiento. }\end{array}$ \\
\hline
\end{tabular}

Fuente: [45,46].

\section{Conclusiones}

La revisión de la literatura permite concluir que la gestión de la cooperación en proyectos de software libre, aunque posible, no ha sido abordada con la suficiente intensidad para proveer a la comunidad de desarrolladores de reglas y estrategias que permitan una acción colectiva efectiva que mejore la calidad, cantidad y permanencia de las aplicaciones desarrolladas bajo el enfoque del software libre.
A pesar de que el trabajo de la comunidad desarrolladora de software libre se realiza por medio de cooperación, y de que en su filosofía está que "la cooperación con los demás contribuye a la base de la sociedad", en esta revisión se encontró que en la actualidad no hay una estrategia ordenada que permita que la productividad de estos grupos y la calidad del software sean óptimas. Se espera que esta revisión permita proponer una estrategia que se pueda adoptar por los grupos desarrolladores 
para que se puedan desarrollar proyectos cada vez más exitosos.

Se justifica realizar una revisión de la literatura en pos de identificar los mecanismos de cooperación que los desarrolladores de software utilizan para enfrentar los dilemas sociales, que pudieran impedir el desarrollo del software libre o, incluso, reducir su disponibilidad. Esta revisión permite encontrar errores cometidos en los modelos o mecanismos actuales, y así poder proponer un mecanismo que puede ser más efectivo, ya que se realiza una evaluación de este.

Se determinó que el mecanismo más adecuado, entrelos revisados, para evaluaruna estrategia que permita promover la cooperación en proyectos de desarrollo de software libre es la dinámica de sistemas, ya que permite realizar modelamiento y hacer seguimiento del comportamiento del proceso, debido a que se pueden tener en cuenta los retardos e ir revisando qué sucede en el momento de la toma de decisiones. Por medio de la dinámica de sistemas se puede ir revisando cómo es el comportamiento de acuerdo con las condiciones iniciales y cómo se afectaría la confianza dependiendo del valor de la confianza inicial. Las demás herramientas podrían dejar inconcluso lo que suceda en el comportamiento del proceso, ya que presentan debilidades en comparación con la dinámica de sistemas.

Es importante que exista una confianza de cooperación que permita que esta se incremente en el futuro y así, finalmente, se pueda promover y mantener la cooperación. También es importante manejar una reputación que se debe ir incrementando; esta es importante porque de ella podría depender que exista una confianza; a mayor reputación, mayor confianza; a mayor confianza, mayor respuesta, y finalmente se obtendrá mayor cooperación.

\section{REFERENCIAS}

[1] P. Kollock, "Social Dilemmas: The Anatomy of Cooperation. Annual Review", Annual Review of Sociology, 1998, pp. 183-214.

[2] E. S. Raymond. "The Cathedral \& the Bazaar. Musings on Linux and Open Source by an Accidental Revolutionary", O'Reilly \& Associates, Inc., 2001, pp. 258.

[3] R.G. Hardin, "The tragedy of the commons. Science", Science, New Series, Vol. 162, No. 3859, pp. 1243-1248, Dec. 13, 1968.

[4] M. Olson, The logic of the collective action: Public Goods and the theory of groups, Harvard University Press: Cambribge. 1965, p. 167.

[5] E. Ostrom, "A behavioral approach to the rational choice theory of collective action". American Political Science Review, vol. 92, No. 1, 1998, pp. 1-22.

[6] J. A. Parra, Constructo para la evaluación de la cooperación en dilemas sociales de gran escala (Tesis doctoral en Ingeniería-Sistemas)”, Ed. Universidad Nacional de Colombia, Sede Medellín. 2011, p. 283.

[7] E. Ostrom, Governing the commons: The evolution of institutions for collective Action, Cambridge University Press,1990, pp. 280.

[8] E. Ostrom \& J. Walker, Trust and reciprocity: Interdisciplinary lessons from experimental research, Russell Sage Foundation Publications, 2005, pp. 424.

[9] J. Walker \& E. Ostrom, "Trust and reciprocity as foundations for cooperation: Individuals, institutions, and context", In Capstone Meeting of the RSF Trust Initiative at the Russell Sage Foundation, May, 2007.

[10] E. Ostrom, "A behavioral approach to the rational choice theory of collective action". In Polycentric games and institutions: readings 
from the Workshop in Political Theory and Policy Analysis, University of Michigan Press, 2000, pp. 472.

[11] L. Mark'oczy, Trust but verify: Distinguishing distrust from vigilance. Presentado en la Academy of Management Conference en Seattle, 2003.

[12] R. Luce, H. Raiffa \& T. Teichmann, "Games and decisions". Physics Today, pp. 11, 33, 1958.

[13] M. Olson, The logic of collective action: Public goods and the theory of groups. Harvard University Press. 1971.

[14] D. Castillo \& A. Saysel, "Simulation of common pool resource field experiments: a behavioral model of collective action", Ecological Economics, 55 (3), pp. 420-436, 2005.

[15] R. Smith, "Resolving the tragedy of the commons by creating private property rights in wildlife", Cato Journal, 1 (2), pp.439-468, 1981.

[16] G.D. Martner, Gobernar el mercado. Las nuevas fronteras del Estado en el siglo XXI, Santiago de Chile: LOM, 1999.

[17] J. A. Parra, "Gestión de la Cooperación en Dilemas Sociales: Un Aporte Desde la Ingeniería de Sistemas", presentado en el Primer Congreso Internacional y Nacional en Innovación en Gestión, Universidad Francisco de Paula Santander, Ocaña, Colombia, 2012.

[18] P. Johnson, “Agent-based modeling”. Social Science Computer Review, 20 (2), pp.174, 2002.

[19] M. Janssen \& E. Ostrom, "Empirically based, agent-based models". Ecology and Society, 11 (2), 37, 2006.

[20] R. Sawyer, "Social emergence: Societies as complex systems", Cambridge University Press, 2005.
[21] R. Conte, B. Edmonds, S. Moss \& R. Sawyer, "Sociology and social theory in agent based social simulation: A symposium", Computational \& Mathematical Organization Theory, 7(3), pp.183-205, 2001.

[22] S. Wolfram, "Cellular automata. Modeling Chemical Systems Using Cellular Automata", pp. 9-38, 2005.

[23] V. Akimov \& M. Soutchanski, "Automata simulation of n-person social dilemma games", Journal of Conflict Resolution, 38 (1), pp.138-148, 1994.

[24] S. Wolfram, "Universality and complexity in cellular automata", Physica D: Nonlinear Phenomena, 10 (1-2), pp.1-35, 1984.

[25] M. Davis, Game theory: a nontechnical introduction, Dover Pubns. 1997.

[26] M. Osborne \& A. Rubinstein, A course in game theory, The MIT Press, 1994.

[27] R. Gibbons, A primer in game theory, FT Prentice Hall, 1992.

[28] R. Axelrod \& W. Hamilton, "The evolution of cooperation”, Science, 211 (4489), pp. 1390, 1981.

[29] C. Nemeth, "A critical analysis of research utilizing the prisoners dilemma paradigm for the study of bargaining", Advances in Experimental Social Psychology, 6, pp. 203234, 1972.

[30] R. Myerson, Game theory: analysis of conflict, Harvard University Press, 1997.

[31] E. Diehl \& J. Sterman, "Effects of feedback complexity on dynamic decision making", Organizational Behavior and Human Decision Processes, 62 (2), pp. 198-215, 1995.

[32] E. Rasmusen, Games and Information: An Introduction to Game Theory, WileyBlackwell, 2007. 
[33] J. Sterman, "Misperceptions of feedback in dynamic decision making", Organizational behavior and Human Decision Processes, 43 (3), pp. 301-335, 1989.

[34] System Dynamics Society, System Dynamics Society, 2010.

[35] Y. Barlas, "Formal aspects of model validity and validation in system dynamics", System Dynamics Review, 12 (3), pp. 183-210, 1996.

[36] J. Morecroft, Strategic Modelling and Business Dynamics: a Feedback Systems Approach, Wiley, 2007.

[37] J. Forrester, Industrial Dynamics, MIT Press Cambridge, MA, 1961.

[38] J. Forrester, "Policies, Decisions and Information Sources for Modeling", European Journal of Operational Research, 59 (1), pp. 42-63, 1992.

[39] J. Morecroft, "System dynamics: Portraying Bounded Rationality", Omega, 11 (2), pp. 131-142, 1983.
[40] J. Bell, \& P. Senge, "Methods for enhancing refutability in system dynamics modeling", System Dynamics Review, 14, pp. 61-73, 1980.

[41] J. Sterman, Business dynamics: Systems thinking and modeling for a complex world with CD-ROM, Irwin/McGraw-Hill, 2000.

[42] R. M. Stallman "Software libre para una sociedad libre", versión 1.0, Diciembre de 2004, pp. 232.

[43] P. M. Osterloh, "Open Source-New Rules in Software Development”, pp. 1-23. 2001.

[44] M. Osterloh \& S. Rota, Trust and Community in Open Source Software Production, 2004.

[45] M. Vidal, Cooperación sin mando: una introducción al software libre", pp. 1-13, 2000 .

[46] E. S. Raymond, The Cathedral \& the Bazaar. Musings on Linux and Open Source by an Accidental Revolutionary, 2001. 